\title{
STABILITY THEOREMS FOR CONFORMAL FOLIATIONS
}

\author{
ROBERT A. BLUMENTHAL
}

\begin{abstract}
The global stability theorem of G. Reeb is no longer true if the codimension of the foliation is greater than one. However, in the presence of a complete transverse Riemannian structure, B. Reinhart obtained a global stability result. We prove global stability theorems for the much larger class of conformal foliations.
\end{abstract}

1. Introduction. An important problem in foliation theory is the study of the influence exerted by a compact leaf upon the global structure of a foliation. For certain classes of foliations, this influence is considerable.

TheOREM (ReEB StABiLITy [7]). Let $\mathscr{F}$ be a codimension one foliation of a compact connected manifold $M$. If $\mathscr{F}$ has a compact leaf with finite fundamental group, then all the leaves of $\mathscr{F}$ are compact with finite fundamental group.

This theorem is false for foliations of codimension greater than one. However, in the presence of a certain transverse geometric structure one has the following global stability result.

TheOREM (ReINHART Stability [8]). Let $\mathscr{F}$ be a complete Riemannian foliation of a connected manifold $M$. Then all the leaves of $\mathscr{F}$ have the same universal covering space. In particular, if F has a compact leaf with finite fundamental group, then all the leaves of $\mathscr{F}$ are compact with finite fundamental group.

Recall that a codimension $q$ foliation $\mathscr{F}$ of a manifold $M$ is conformal if the frame bundle of its normal bundle admits a foliate reduction to the conformal group $\operatorname{CO}(q)$ or, equivalently, if its normal bundle admits a basic connection whose holonomy group is contained in $\mathrm{CO}(q)$ [11]. Equivalently, $\mathscr{F}$ admits a transverse $\mathrm{CO}(q)$-structure in the sense of [1]. In $\$ 2$ we consider foliations which admit a transverse $G$-structure of finite type and completeness of such foliations and we prove

THEOREM 1. Let $\mathscr{F}$ be a complete conformal foliation of codimension $q \geqslant 3$ of a connected manifold $M$. Then all the leaves of $\mathscr{F}$ have the same universal covering space.

Received by the editors September 27, 1982. Presented to the Society, January 5, 1983 at the 89th Annual Meeting in Denver.

1980 Mathematics Subject Classification. Primary 57R30; Secondary 53C10, 53A30.

Key words and phrases. Conformal foliation, $G$-foliation, infinitesimal holonomy groups.

(C)1984 American Mathematical Society $0002-9939 / 84 \$ 1.00+\$ .25$ per page 
Stability theorems often involve some finiteness condition on the holonomy of a compact leaf. The local stability theorem of Reeb [7] is of this type and in [5] it is shown that if $\mathscr{F}$ is a complete Riemannian foliation possessing a compact leaf with finite linear holonomy group, then all the leaves of $\mathscr{F}$ are compact with finite linear holonomy group. If $L$ is a leaf of a foliation, we denote by $h^{r}(L, x)$ the infinitesimal holonomy group of order $r$ of $L$ at $x \in L$. Of course, $h^{1}(L, x)$ is just the linear holonomy group of $L$. In $\S 3$ we prove

THEOREM 2. Let $\mathscr{F}$ be a complete conformal foliation of codimension $q \geqslant 3$ of a connected manifold $M$. If $\mathscr{F}$ has a compact leaf $L_{0}$ with $h^{2}\left(L_{0}, x\right)$ finite, then all the leaves of $\mathscr{F}$ are compact and $h^{2}(L, x)$ is finite for all $L \in \mathscr{F}$.

\section{Prolongations of transverse $G$-structures.}

(2.1) Definition. Let $(M, \mathscr{F})$ be a foliated manifold, codim $\mathscr{F}=q$. A transverse parallelism on $(M, \mathscr{F})$ is a collection of vector fields $\left\{X_{1}, \ldots, X_{q}\right\}$ on $M$ which at each point $x$ of $M$ spans a subspace of $T_{x}(M)$ complementary to the subspace tangent to $\mathscr{F}$ and satisfies $\left[Y, X_{i}\right]$ is tangent to $\mathscr{F}$ whenever $Y$ is tangent to $\mathscr{F}$ for $i=1, \ldots, q$. A complete transverse parallelism on $(M, \mathscr{F})$ is a transverse parallelism $\left\{X_{1}, \ldots, X_{q}\right\}$ on $(M, \mathscr{F})$ such that each $X_{i}$ is a complete vector field on $M$. We say that $(M, \mathscr{F})$ is transversely parallelizable (respectively, completely transversely parallelizable) if it admits a transverse parallelism (respectively, complete transverse parallelism).

(2.2) LEMMA [6]. Let $(M, \mathscr{F})$ be completely transversely parallelizable with $M$ connected. Then the group $\operatorname{Aut}(M, \mathscr{F})$ of diffeomorphisms of $M$ which preserve $\mathscr{F}$ acts transitively on $M$.

Proof. We first remark that if $L$ is a leaf of $\mathscr{F}$, then for any $p, q \in L$ there exists $\phi \in \operatorname{Aut}(M, \mathscr{F})$ such that $\phi(p)=q$. Let $X_{1}, \ldots, X_{q}$ be a complete transverse parallelism on $(M, \mathscr{F})$. For $i=1, \ldots, q$ let $\phi^{i}: \mathbf{R} \times M \rightarrow M$ be the action of $\mathbf{R}$ on $M$ generated by $X_{i}$. Then $\phi_{t}^{i} \in \operatorname{Aut}(M, \mathscr{F})$ for all $t \in \mathbf{R}, i=1, \ldots, q$. Let $p \in M$. Define $\Phi: \mathbf{R}^{q} \rightarrow \mathbf{M}$ by

$$
\Phi\left(t_{1}, \ldots, t_{q}\right)=\phi_{t_{1}}^{1} \circ \phi_{t_{2}}^{2} \circ \cdots \circ \phi_{t_{q}}^{q}(p) .
$$

Let $f: U \rightarrow \mathbf{R}^{q}$ be a submersion constant along the leaves of $\mathscr{F} \mid U$, where $U$ is a neighborhood of $p \in M$. Since $\Phi_{*_{0}}\left(\partial /\left.\partial x^{i}\right|_{0}\right)=X_{i_{p}}$ for $i=1, \ldots, q$, it follows that $(f \circ \Phi)_{*_{0}}: T_{0}\left(\mathbf{R}^{q}\right) \rightarrow T_{f(p)}\left(\mathbf{R}^{q}\right)$ is an isomorphism. Hence there is a neighborhood $V$ of 0 in $\mathbf{R}^{q}$ and a neighborhood $W$ of $f(p)$ in $\mathbf{R}^{q}$ such that $\Phi(V) \subset U$ and $f \circ \Phi$ : $V \rightarrow W$ is a diffeomorphism. Hence $\Phi: V \rightarrow M$ is an imbedded submanifold transverse to $\mathscr{F}$. Let $L$ be any leaf in the saturation of $\Phi(V)$. Let $q \in L \cap \Phi(V)$. There is a point $\left(t_{1}, \ldots, t_{q}\right) \in V$ such that $q=\Phi\left(t_{1}, \ldots, t_{q}\right)$. Thus $q=\phi_{t_{1}}^{1} \circ \ldots$ - $\phi_{t_{4}}^{q}(p)$ and hence $q$ is in the orbit of $p$ under $\operatorname{Aut}(M, \mathscr{F})$. By the earlier remark, $L$ is contained in this orbit. Thus the saturation of $\Phi(V)$ is contained in the orbit of $p$. Since this saturation is open, we have that the orbit of $p$ is all of $M$.

We now construct prolongations of a transverse $G$-structure and, in particular, the first prolongation of a conformal foliation. 
Let $\mathscr{F}$ be a smooth codimension $q$ foliation of the connected manifold $M$. Let $E \subset T(M)$ be the tangent bundle of $\mathscr{F}$ and let $Q=T(M) / E$ be the normal bundle of $\mathscr{F}$. Let $\rho: F(Q) \rightarrow M$ be the frame bundle of $Q$, a principal GL $(q, \mathbf{R})$-bundle. Let $G$ be a Lie subgroup of $\operatorname{GL}(q, \mathbf{R})$. Let $P$ be a transverse $G$-structure on $(M, \mathscr{F})$. That is, $P \subset F(Q)$ is a reduction to a principal $G$-bundle such that the natural parallelism along the leaves of $\mathscr{F}$ carries elements of $P$ to elements of $P$ [1]. Note that $\mathscr{F}$ is a $G$-foliation in the sense of [3]. There is a smooth $q$-dimensional manifold $N$ and an $N$-cocycle $\left\{\left(U_{\alpha}, f_{\alpha}, g_{\alpha \beta}\right)\right\}_{\alpha, \beta \in A}$ defining $\mathscr{F}$ such that the $g_{\alpha \beta}$ 's are local automorphisms of a $G$-structure $R \subset L(N)$ on $N$, where $L(N)$ denotes the bundle of linear frames over $N$. Then $\left\{\left(\rho^{-1}\left(U_{\alpha}\right), f_{\alpha_{*}}, g_{\alpha \beta_{*}}\right)\right\}_{\alpha, \beta \in A}$ is an $R$-cocycle on $P$ and hence defines a foliation $\mathscr{F}_{0}$ of $P$ with $\operatorname{dim} \mathscr{F}_{0}=\operatorname{dim} \mathscr{F}_{\text {. If }} L_{0} \in \mathscr{F}_{0}$; and $L=\rho\left(L_{0}\right)$ $\in \mathscr{F}$, then $\left.\rho\right|_{L_{0}}: L_{0} \rightarrow L$ is a regular covering.

Let $\mathfrak{g}$ be the Lie algebra of $G$. Let $k \in\{0,1,2, \ldots\}$. The $k$ th prolongation $\mathfrak{g}_{k}$ of $\mathfrak{g}$ is the space of symmetric multilinear mappings

$$
t: \underbrace{\mathbf{R}^{q} \times \cdots \times \mathbf{R}^{q}}_{(k+1) \text {-times }} \rightarrow \mathbf{R}^{q}
$$

such that, for each fixed $v_{1}, \ldots, v_{k} \in \mathbf{R}^{q}$, the linear transformation of $\mathbf{R}^{q}$ given by $v \rightarrow t\left(v, v_{1}, \ldots, v_{k}\right)$ belongs to $\mathrm{g}$. The $k$ th prolongation $G_{k}$ of $G$ is the group of linear transformations $\bar{t}$ of $V \oplus \mathrm{g} \oplus \mathrm{g}_{1} \oplus \cdots \oplus \mathrm{g}_{k-1}$ of the form

$$
\begin{aligned}
& \bar{t}(x)=x \quad \text { for } x \in \mathfrak{g} \oplus \mathfrak{g}_{1} \oplus \cdots \oplus \mathfrak{g}_{k-1}, \\
& \bar{t}(v)=v+t(\cdot, \ldots, \cdot, v) \text { for } v \in V
\end{aligned}
$$

where $t \in \mathfrak{g}_{k}[4]$ (also cf. [10, 9]).

Let $V=\mathbf{R}^{q}$. As in [4], define $\mathrm{\partial}: \mathfrak{g} \otimes V^{*} \rightarrow V \otimes \wedge^{2} V^{*}$ by

$$
(\partial f)\left(v_{1}, v_{2}\right)=-f\left(v_{2}\right) v_{1}+f\left(v_{1}\right) v_{2}, \quad f \in \mathfrak{g} \otimes V^{*}, v_{1}, v_{2} \in V .
$$

Let $C \subset V \otimes \wedge^{2} V^{*}$ be a subspace satisfying $V \otimes \Lambda^{2} V^{*}=\partial\left(g \otimes V^{*}\right) \oplus C$. In general there is no natural choice of $C$.

Let $\theta$ be the canonical form on $P$. It is the $V$-valued one-form on $P$ defined by

$$
\theta_{u}(Y)=u^{-1}\left(\pi \rho_{*_{u}}(Y)\right), \quad u \in P, Y \in T_{u}(P),
$$

where $\pi: T(M) \rightarrow Q$ is the natural bundle projection and $u: V \rightarrow Q_{\rho(u)}$ denotes the vector space isomorphism which sends the standard basis of $V$ to the frame $u$ of $Q_{\rho(u)}$. Let $E_{0} \subset T(P)$ be the subbundle tangent to $\mathscr{F}_{0}$. Then $Q_{0}=T(P) / E_{0}$ is the normal bundle of $\mathscr{F}_{0}$.

Let $u \in P$. Then $\theta_{u}: T_{u}(P) \rightarrow V$ induces a map $\theta_{u}: Q_{0_{u}} \rightarrow V$. A $q$-dimensional subspace $H$ of $Q_{0_{u}}$ is called horizontal if $\theta_{u}: H \rightarrow V$ is an isomorphism. Since $i(X) d \theta=0$ for all vector fields $X$ tangent to $\mathscr{F}_{0}$, it follows that $(d \theta)_{u}: T_{u}(P) \times$ $T_{u}(P) \rightarrow V$ induces a skew-symmetric bilinear mapping $(d \theta)_{u}: Q_{0_{u}} \times Q_{0_{u}} \rightarrow V$. To each horizontal subspace $H \subset Q_{0_{u}}$ we associate $c(u, H) \in V \otimes \Lambda^{2^{u}} V^{*}$ by restricting $(d \theta)_{u}: Q_{0_{u}} \times Q_{0_{u}} \rightarrow V$ to $H \times H$ and identifying $H$ with $V$ via $\theta_{u}$.

Let $u \in P$ and let $H \subset Q_{0_{u}}$ be a horizontal subspace. Define $z_{H}: V \oplus \mathfrak{g} \rightarrow Q_{0_{u}}$ by

$$
z_{H}(v \oplus A)=\theta_{u}^{-1}(v)+\pi\left(A_{u}^{*}\right),
$$


where $A^{*}$ is the fundamental vector field on $P$ induced by $A \in \mathfrak{g}$ and $\pi: T_{u}(P) \rightarrow Q_{0_{u}}$ is the natural bundle projection. Then $z_{H}$ is a linear isomorphism and hence may be regarded as a linear frame of $Q_{0_{u}}$. Let $F\left(Q_{0}\right)$ be the frame bundle of $Q_{0}$. We define the first prolongation $P_{1}$ of $\left(P, \mathscr{F}_{0}\right)$ by

$$
P_{1}=\left\{z_{H} \in F\left(Q_{0}\right): H \subset Q_{0_{u}} \text { is horizontal, } u \in P, c(u, H) \in C\right\} .
$$

Then, by an argument similar to that in [4], $P_{1}$ is a transverse $G_{1}$-structure on $\left(P, \mathscr{F}_{0}\right)$. Note that $P_{1}$ carries a foliation $\mathscr{F}_{1}$ whose leaves are coverings of the leaves of $\mathscr{F}_{0}$. We define the $k$ th prolongation $\left(P_{k}, \mathscr{F}_{k}\right)$ of $\left(P, \mathscr{F}_{0}\right)$ inductively by setting $\left(P_{k}, \mathscr{F}_{k}\right)=\left(\left(P_{k-1}\right)_{1},\left(\mathscr{F}_{k-1}\right)_{1}\right)$, the first prolongation of $\left(P_{k-1}, \mathscr{F}_{k-1}\right)$. Then $\left(P_{k}, \mathscr{F}_{k}\right)$ is a transverse $G_{k}$-structure on $\left(P_{k-1}, \mathscr{F}_{k-1}\right)$.

For the remainder of this section we assume that $\mathscr{F}$ is a conformal foliation of $M$ with $q \geqslant 3$. Thus

$$
\begin{aligned}
& G=\operatorname{CO}(q)=\left\{A \in \mathrm{GL}(q, \mathbf{R}):{ }^{t} A A=c I, c \in \mathbf{R}, c>0\right\}, \\
& \mathrm{g}=\operatorname{co}(q)=\left\{A \in \operatorname{gl}(q, \mathbf{R}):{ }^{t} A+A=c I, c \in \mathbf{R}\right\} .
\end{aligned}
$$

Note that since the kernel of $\partial$ is isomorphic to $\mathfrak{g}_{1}$ and $\operatorname{dim} \mathfrak{g}_{1}=q[4]$, we have

$\operatorname{dim} \operatorname{image}(\partial)=\operatorname{dim} g \otimes V^{*}-\operatorname{dim} \operatorname{kernel}(\partial)$

$$
=(q(q-1) / 2+1) q-q=q^{2}(q-1) / 2=\operatorname{dim} V \otimes \wedge^{2} V^{*},
$$

so $\partial$ is onto. Thus the only choice of $C$ is $C=\{0\}$, whence $\left(P_{1}, \mathscr{F}_{1}\right)$ is canonically defined. Since $g_{2}=\{0\}[4]$, we have that $\left(P_{2}, \mathscr{F}_{2}\right)$ is a transverse $\{1\}$-structure on $\left(P_{1}, \mathscr{F}_{1}\right)$ so $\left(P_{1}, \mathscr{F}_{1}\right)$ is transversely parallelizable. This is false for $q=1,2$ since $\operatorname{co}(q)$ is of infinite type if $q=1,2$.

(2.3) Definition. We say $\mathscr{F}$ is a complete conformal foliation of $M$ if $\left(P_{1}, \mathscr{F}_{1}\right)$ is completely transversely parallelizable.

REMARK. If $\mathscr{F}$ is a Riemannian foliation of $M$ (i.e., $G=O(q)$ ), then $\mathrm{g}_{1}=\{0\}$ and so $\left(P, \mathscr{F}_{0}\right)$ is transversely parallelizable. If $\mathscr{F}$ is a complete Riemannian foliation of $M$, then $\left(P, \mathscr{F}_{0}\right)$ is completely transversely parallelizable.

Without loss of generality we may assume (by passing to a finite cover of $M$ if necessary) that $P$, and hence $P_{1}$, is connected. If $\mathscr{F}$ is a complete conformal foliation of $M$ with $q \geqslant 3$, then $\operatorname{Aut}\left(P_{1}, \mathscr{F}_{1}\right)$ acts transitively on $P_{1}$ by Lemma (2.2) and so all the leaves of $\mathscr{F}_{1}$ are diffeomorphic. Since the leaves of $\mathscr{F}_{1}$ are coverings of the leaves of $\mathscr{F}$, it follows that all the leaves of $\mathscr{F}$ have the same universal covering space.

3. Infinitesimal holonomy groups. Let $\mathscr{F}$ be a foliation of a manifold $M$ and let $L$ be a leaf of $\mathscr{F}$. Recall (see e.g. [2]) the definitions of the holonomy group and infinitesimal holonomy groups of $L$. Fix $x_{0} \in L$. Let $\sigma:[0,1] \rightarrow L$ be a loop at $x_{0}$. Choose a covering of $\sigma$ by a finite sequence of open sets $U_{0}, U_{1}, \ldots, U_{n-1}, U_{n}=U_{0}$ with $x_{0} \in U_{0}, \quad U_{i} \cap U_{i+1} \neq \varnothing$ for $i=0,1, \ldots, n-1$ such that, for each $i=$ $0,1, \ldots, n, f_{i}: U_{i} \rightarrow \mathbf{R}^{q}(q=\operatorname{codim} \mathscr{F})$ is a submersion defining $\mathscr{F} \mid U_{i}, f_{0}=f_{n}$, $f_{0}\left(x_{0}\right)=0$. For each $i=0,1, \ldots, n-1$ there is a diffeomorphism $g_{i+1, i}$ : $f_{i}\left(U_{i} \cap U_{i+1}\right) \rightarrow f_{i+1}\left(U_{i} \cap U_{i+1}\right)$ such that $f_{i+1}=g_{i+1, i} \circ f_{i}$ on $U_{i} \cap U_{i+1}$. Then $g_{n, n-1} \circ \cdots \circ g_{1,0}$ is a local diffeomorphism defined in a neighborhood of 0 in $\mathbf{R}^{q}$ fixing 0 . Let $h(\sigma)$ be its germ at 0 . Since $h(\sigma)$ depends only on the homotopy class 
of $\sigma$, we obtain a homomorphism $h: \pi_{1}\left(L, x_{0}\right) \rightarrow G(q)$, well-defined up to conjugacy, where $G(q)$ denotes the group of germs of local diffeomorphisms of $\mathbf{R}^{q}$ fixing 0 . The holonomy group of $L$ based at $x_{0}$ is defined by $h\left(L, x_{0}\right)=\operatorname{image}(h) \subset$ $G(q)$. A change of basepoint produces a conjugate subgroup. Let $G^{r}(q)$ be the group of $r$-jets at $0 \in \mathbf{R}^{q}$ and let $\pi^{r}: G(q) \rightarrow G^{r}(q)$ be the natural projection. The infinitesimal holonomy group of order $r$ of $L$ at $x_{0}$ is defined by $h^{r}\left(L, x_{0}\right)=$ image $\left(h^{r}\right) \subset G^{r}(q)$ where $h^{r}=\pi^{r} \circ h$.

The following construction gives a useful realization of $h^{r}\left(L, x_{0}\right)$. Let $U$ and $V$ be neighborhoods of 0 in $\mathbf{R}^{q}$ and let $f: U \rightarrow M, g: V \rightarrow M$ be smooth maps transverse to $\mathscr{F}$ with $f(0)=g(0)=x$. Let $W$ be a neighborhood of $x$ and let $F: W \rightarrow \mathbf{R}^{q}$ be a submersion defining $\mathscr{F} \mid W$. We say that $f$ and $g$ define the same transverse $r$-frame at $x$ if $F \circ f$ and $F \circ g$ define the same $r$-frame at $F(x)$, i.e., if $F \circ f$ and $F \circ g$ have the same partial derivatives up to order $r$ at 0 . This definition is independent of the choice of the distinguished function $F$. The transverse $r$-frame determined by $f$ is denoted by $j_{x}^{r}(f)$. Let $P^{r}(M, \mathscr{F})$ be the set of transverse $r$-frames on $M$. Then $\pi_{r}$ : $\operatorname{Pr}^{r}(M, \mathscr{F}) \rightarrow M, \pi_{r}\left(j_{x}^{r}(f)\right)=x$ is a principal bundle over $M$ with group $G^{r}(q)$. The right action of $G^{r}(q)$ on $P^{r}(M, \mathscr{F})$ is given by $j_{x}^{r}(f) j_{0}^{r}(g)=j_{x}^{r}(f \circ g)$ for $j_{x}^{r}(f) \in$ $\operatorname{Pr}^{r}(M, \mathscr{F}), j_{0}^{r}(g) \in G^{r}(q)$. Clearly $P^{1}(M, \mathscr{F})$ is the bundle $F(Q)$ of linear frames of the normal bundle of $\mathscr{F}$ with group $G^{1}(q)=\mathrm{GL}(q, \mathbf{R})$. Let $F: W \rightarrow \mathbf{R}^{q}$ be a submersion defining $\mathscr{F} \mid W$. Then $F$ induces a submersion $F^{(r)}: P^{r}(M, \mathscr{F}) \mid W \rightarrow$ $P^{r}\left(\mathbf{R}^{q}\right)$ by $F^{(r)}\left(j_{x}^{r}(f)\right)=j_{F(x)}^{r}(F \circ f)$, where $P^{r}\left(\mathbf{R}^{q}\right)$ denotes the bundle of $r$-frames of $\mathbf{R}^{q}$. Note that $P^{r}(M, \mathscr{F}) \mid W=F^{-1}\left(P^{r}\left(\mathbf{R}^{q}\right) \mid F(W)\right)$. Let $\left\{\left(W_{\alpha}, F_{\alpha}, h_{\alpha \beta}\right)\right\}_{\alpha, \beta \in A}$ be an $\mathbf{R}^{q}$-cocycle defining $\mathscr{F}$. Then $\left\{\left(\pi_{r}^{-1}\left(W_{\alpha}\right), F_{\alpha}^{(r)}, h_{\alpha \beta}^{(r)}\right)\right\}_{\alpha, \beta \in A}$ is a $P^{r}\left(\mathbf{R}^{q}\right)$-cocycle on $P^{r}(M, \mathscr{F})$ and hence defines a foliation $\mathscr{F}^{r}$ of $P^{r}(M, \mathscr{F})$ with $\operatorname{dim} \mathscr{F}^{r}=\operatorname{dim} \mathscr{F}$. Let $L \in \mathscr{F}$. Then $\operatorname{Pr}^{r}(M, \mathscr{F}) \mid L$ has a canonical flat connection whose holonomy group is isomorphic to $h^{r}\left(L, x_{0}\right)$. Let $L^{r} \in \mathscr{F}^{r}$ be a leaf such that $\pi_{r}\left(L^{r}\right)=L$. Then $\pi_{r}: L^{r} \rightarrow L$ is a regular covering whose group of covering transformations is isomorphic to $h^{r}\left(L, x_{0}\right)$.

(3.1) LemMA. The first prolongation of $P^{1}(M, \mathscr{F})$ is $P^{2}(M, \mathscr{F})$; i.e., $P^{1}(M, \mathscr{F})_{1}=$ $P^{2}(M, \mathscr{F})$.

Proof. We first remark that the natural map $P^{2}(M, \mathscr{F}) \rightarrow P^{1}(M, \mathscr{F})$ is a principal $N_{1}^{2}(q)$-bundle, where $N_{1}^{2}(q)$ is the kernel of the natural map $G^{2}(q) \rightarrow$ $G^{1}(q)$. Let $j_{x}^{2}(f) \in P^{2}(M, \mathscr{F})$. Then $f: U \rightarrow M$ where $U$ is a neighborhood of 0 in $\mathbf{R}^{q}, f(0)=x$, with $f$ transverse to $\mathscr{F}$. Define $\bar{f}: P^{1}\left(\mathbf{R}^{q}\right) \mid U \rightarrow P^{1}(M, \mathscr{F})$ by $\bar{f}\left(j_{y}^{1}(g)\right)$ $=j_{f(y)}^{1}(f \circ g)$. Then $\bar{f}$ is transverse to $\mathscr{F}^{1}$. Let $e=j_{0}^{1}(\mathrm{Id}) \in P^{1}\left(\mathbf{R}^{q}\right)$ and let $Q^{1}$ be the

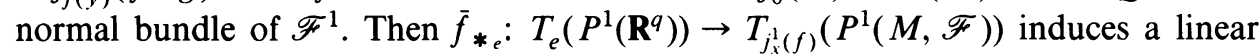
isomorphism $\bar{f}_{*_{e}}: T_{e}\left(P^{1}\left(\mathbf{R}^{q}\right)\right) \rightarrow Q_{j_{k}^{\mathrm{l}}(f)}^{1}$. This linear isomorphism determines a frame $z$ of $Q_{j_{x}^{1}(f)}^{1}$.

Let $\bar{H} \subset T\left(P^{1}\left(\mathbf{R}^{q}\right)\right)$ be the horizontal distribution corresponding to the canonical flat linear connection on $\mathbf{R}^{q}$ and let $\bar{\theta}$ be the canonical $V$-valued one-form on $P^{1}\left(\mathbf{R}^{q}\right)$. Let $H=\bar{f}_{*_{e}}\left(\bar{H}_{e}\right) \subset Q_{j_{x}^{1}}^{1}(f)$. Then $H$ is a horizontal subspace of $Q_{j_{x}^{\prime}(f)}^{1}$. Clearly $z=z_{H}$. Since $d \bar{\theta}: \bar{H}_{e} \times \bar{H}_{e} \rightarrow V$ is zero (the canonical flat connection on $\mathbf{R}^{q}$ has zero torsion) and since $\bar{f}^{*}(\theta)=\bar{\theta}$, we have that $d \theta: H \times H \rightarrow V$ is zero and so 
$c\left(j_{x}^{1}(f), H\right)=0$. Since the only choice of $C$ for $G=\mathrm{GL}(\mathrm{q}, \mathbf{R})$ is $C=\{0\}$, we have that $z=z_{H} \in P^{1}(M, \mathscr{F})_{1}$. Thus the correspondence $j_{x}^{2}(f) \rightarrow z$ defines a map $P^{2}(M, \mathscr{F}) \rightarrow P^{1}(M, \mathscr{F})_{1}$.

We show that this map is an isomorphism between the bundles $P^{2}(M, \mathscr{F}) \rightarrow$ $P^{1}(M, \mathscr{F})$ and $P^{1}(M, \mathscr{F})_{1} \rightarrow P^{1}(M, \mathscr{F})$ by exhibiting a compatible isomorphism between the groups $N_{1}^{2}(q)$ and $\operatorname{GL}(q, \mathbf{R})_{1}$. Let $j_{0}^{2}(g) \in N_{1}^{2}(q)$. There is a unique polynomial representation

$$
g^{i}\left(y^{1}, \ldots, y^{q}\right)=\sum a_{j}^{i} y^{j}+\sum a_{j k}^{i} y^{j} y^{k}, \quad i=1, \ldots, q,
$$

where the coefficients $a_{j k}^{i}$ are symmetric in the lower indices. Since $j_{0}^{1}(g)=0$, we have $a_{j}^{i}=0$ for $i, j=1, \ldots, q$. Thus $j_{0}^{2}(g)$ can be uniquely represented by the $q$ symmetric $q \times q$ matrices $\left(a_{j k}^{1}\right),\left(a_{j k}^{2}\right), \ldots,\left(a_{j k}^{q}\right)$. Since $\mathrm{GL}(q, \mathbf{R})_{1}$ is naturally isomorphic to $\operatorname{gl}(q, \mathbf{R})_{1}=$ the space of symmetric bilinear mappings $V \times V \rightarrow V$, the desired isomorphism of the two groups is immediate.

(3.2) LeMma. Let $\mathscr{F}$ be a codimension $q$ conformal foliation of $M$ and let $P$ be a transverse $\mathrm{CO}(q)$-structure on $(M, \mathscr{F})$. Let $p: P_{1} \rightarrow M$ be the composition of the bundle projections $P_{1} \rightarrow P, P \rightarrow M$. Let $L$ be a leaf of $\mathscr{F}$ and let $L_{1}$ be a leaf of $\mathscr{F}_{1}$ such that $p\left(L_{1}\right)=L$. Then $p: L_{1} \rightarrow L$ is a regular covering whose group of covering transformations is isomorphic to $h^{2}\left(L, x_{0}\right)$.

Proof. We define a bundle injection

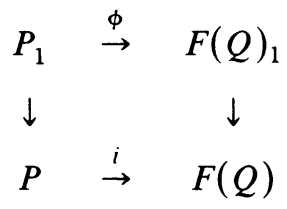

as follows. Let $i=P \rightarrow F(Q)$ be the inclusion map. We map $\operatorname{CO}(q)_{1}$ into $\operatorname{GL}(q, \mathbf{R})_{1}$ by

$$
\mathrm{CO}(q)_{1} \rightarrow \operatorname{co}(q)_{1} \stackrel{j}{\rightarrow} \mathrm{gl}(q, \mathbf{R})_{1} \rightarrow \mathrm{GL}(q, \mathbf{R})_{1}
$$

where $j$ is the inclusion map and the other two arrows are the canonical isomorphisms. Let $\nu\left(P, \mathscr{F}_{0}\right)\left(\right.$ respectively, $\left.\nu\left(F(Q), \mathscr{F}_{0}\right)\right)$ be the normal bundle of $\left(P, \mathscr{F}_{0}\right)$ (respectively, $\left.\left(F(Q), \mathscr{F}_{0}\right)\right)$. Let $u \in P$. Let $H$ be a horizontal subspace of $\nu\left(P, \mathscr{F}_{0}\right)_{u}$ such that $c(u, H)=0$ and let $z_{H}$ be the corresponding element of $P_{1}$. Now $i$ : $P \rightarrow F(Q)$ induces $i_{*}: \nu\left(P, \mathscr{F}_{0}\right)_{u} \rightarrow \nu\left(F(Q), \mathscr{F}_{0}\right)_{u}$. Let $H^{\prime}=i_{*_{u}}(H)$. Then $H^{\prime}$ is a horizontal subspace of $\nu\left(F(Q), \mathscr{F}_{0}\right)_{u}$ satisfying $c\left(u, H^{\prime}\right)=0$. Let $z_{H^{\prime}}$ be the corresponding element of $F(Q)_{1}$. The correspondence $z_{H} \rightarrow z_{H^{\prime}}$ is then the desired map $\phi: P_{1} \rightarrow F(Q)_{1}$.

Since $F(Q)_{1}=P^{1}(M, \mathscr{F})_{1}=P^{2}(M, \mathscr{F})$ by Lemma (3.1), we obtain a bundle injection

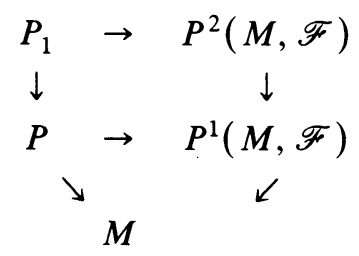


Hence we may regard $p: P_{1} \rightarrow M$ as the restriction to $P_{1}$ of $\pi_{2}: P^{2}(M, \mathscr{F}) \rightarrow M$, and so $p: L_{1} \rightarrow L$ is a regular covering whose group of covering transformations is isomorphic to $h^{2}\left(L, x_{0}\right)$, which completes the proof of the lemma.

Let $\mathscr{F}$ be a complete conformal foliation of codimension $q \geqslant 3$ of a connected manifold $M$. Let $L_{0}$ be a compact leaf of $\mathscr{F}$ with $h^{2}\left(L_{0}, x\right)$ finite. Let $L_{1}$ be a leaf of $\mathscr{F}_{1}$ such that $p\left(L_{1}\right)=L$. By Lemma (3.2) we have that $L_{1}$ is compact. Since $\left(P_{1}, \mathscr{F}_{1}\right)$ is completely transversely parallelizable, it follows from Lemma (2.2) that all leaves of $\mathscr{F}_{1}$ are compact. Also $h^{2}(L, x)$ is finite for all $L \in \mathscr{F}$ by Lemma (3.2) and so Theorem 2 is proved.

(3.3) COROLlaRy. Let $\mathscr{F}$ be a complete conformal foliation of codimension $q \geqslant 3$ of a connected manifold $M$. Then any two leaves of $\mathscr{F}$ without holonomy are diffeomorphic.

Since the germ at a point of a conformal transformation of a $q$-dimensional manifold with $q \geqslant 3$ is determined by the 2-jet of the transformation at that point, we may rephrase Theorem 2 as follows.

THEOREM $2^{\prime}$. Let $\mathscr{F}$ be a complete conformal foliation of codimension $q \geqslant 3$ of a connected manifold $M$. If $\mathscr{F}$ has a compact leaf with finite holonomy group, then all the leaves of $\mathscr{F}$ are compact with finite holonomy group.

\section{REFERENCES}

1. L. Conlon, Transversally parallelizable foliations of codimension two, Trans. Amer. Math. Soc. 194 (1974), 79-102.

2. A. Haefliger, Variétés feuilletées, Ann. Scuola Norm. Sup. Pisa Cl. Sci. (4) 16 (1962), 367-397.

3. F. W. Kamber and P. Tondeur, G-foliations and their characteristic classes, Bull. Amer. Math. Soc. 84 (1978), 1086-1124.

4. S. Kobayashi, Transformation groups in differential geometry, Ergeb. Math. Grenzgeb., vol. 70, Springer-Verlag, Berlin, 1972.

5. P. Molino, Proprietés cohomologiques et proprietés topologiques des feuilletages à connexion transverse projetable. Topology 12 (1973), 317-325.

6. Géométrie globale des feuilletages riemanniens, Proc. Nederl. Akad. Wetensch. A 85 (1982), $45-76$.

7. G. Reeb, Sur certaines propriétés topologiques des variétés feuilletées, Actualités Sci. Indust., no. 1183. Hermann, Paris, 1952.

8. B. Reinhart, Foliated manifolds with bundle-like metrics, Ann. of Math. (2) 69 (1959), 119-132.

9. E. A. Ruh, On the automorphism group of a G-structure, Comment. Math. Helv. 39 (1964), 189-204.

10. S. Sternberg, Lectures on differential geometry, Prentice-Hall, Englewood Cliffs, N.J., 1964.

11. I. Vaisman, Conformal foliations, Kodai Math. J. 2 (1979), 26-37.

Department of Mathematics, Saint Louis University, St. Louis, Missouri 63103 